\title{
The impossibility of a satisfactory population prospect axiology (independently of Finite Fine-Grainedness)
}

\author{
Elliott Thornley ${ }^{1}$
}

Accepted: 21 February 2021/Published online: 11 May 2021

(C) The Author(s) 2021

\begin{abstract}
Arrhenius's impossibility theorems purport to demonstrate that no population axiology can satisfy each of a small number of intuitively compelling adequacy conditions. However, it has recently been pointed out that each theorem depends on a dubious assumption: Finite Fine-Grainedness. This assumption states that there exists a finite sequence of slight welfare differences between any two welfare levels. Denying Finite Fine-Grainedness makes room for a lexical population axiology which satisfies all of the compelling adequacy conditions in each theorem. Therefore, Arrhenius's theorems fail to prove that there is no satisfactory population axiology. In this paper, I argue that Arrhenius's theorems can be repurposed. Since all of our population-affecting actions have a non-zero probability of bringing about more than one distinct population, it is population prospect axiologies that are of practical relevance, and amended versions of Arrhenius's theorems demonstrate that there is no satisfactory population prospect axiology. These impossibility theorems do not depend on Finite Fine-Grainedness, so lexical views do not escape them.
\end{abstract}

Keywords Population axiology · Finite Fine-Grainedness · Arrhenius · Impossibility theorem - Lexical views · Population prospect axiology $\cdot$ Risk · Repugnant conclusion · Population ethics

Elliott Thornley

elliott.thornley@philosophy.ox.ac.uk

1 Faculty of Philosophy, University of Oxford, Radcliffe Observatory Quarter 555, Woodstock Road, Oxford OX2 6GG, UK 


\section{Introduction}

Some possible populations are better than others. For example, a population in which every person lives a wonderful life is better than a population in which those same people live awful lives. What's more, this betterness relation holds (at least sometimes) between populations that differ in size. A population in which every person lives a wonderful life is better than a marginally bigger population in which every person lives an awful life.

These cases are clear-cut, but others are less certain. Is a population in which one million people live a wonderful life better than a population in which one billion people live a good life? Is a population in which two million people live wonderful lives and one million people live awful lives better than a population in which no one lives at all? It would be useful to have a population axiology-a betterness ordering over populations - to adjudicate in cases like these.

Unfortunately, formulating a satisfactory population axiology has proved difficult. Indeed, some claim that it is impossible. Several authors offer impossibility theorems purporting to demonstrate that no population axiology can satisfy a small number of adequacy conditions. ${ }^{1}$ Arrhenius's six theorems represent the state-ofthe-art. ${ }^{2}$ They employ logically weaker and intuitively more compelling adequacy conditions than other theorems extant in the literature, and so have drawn much of the scholarly attention.

However, it has recently been pointed out that each of Arrhenius's six theorems rests on a dubious assumption (Thomas, 2018; Carlson, forthcoming). The assumption, which has been dubbed Finite Fine-Grainedness, states that one can get from a very positive welfare level to a very negative welfare level via a finite number of 'slight' decreases in welfare. ${ }^{3}$ The upshot of denying Finite FineGrainedness is twofold. First, it makes room for a lexical population axiology in which welfare levels and population-values are represented by vectors. Views of this kind constitute a counterexample to Arrhenius's First, Fourth, Fifth, and Sixth Impossibility Theorems. Second, it strips certain adequacy conditions of their plausibility. More precisely, it renders doubtful the Inequality Aversion condition employed in Arrhenius's Second and Third Impossibility Theorems. Therefore, none of Arrhenius's six theorems proves that there is no satisfactory population axiology. Each theorem depends on Finite Fine-Grainedness for the validity of its proof or the plausibility of its adequacy conditions.

Nevertheless, Arrhenius's theorems remain important. In this paper, I demonstrate that they can be turned into theorems stating the impossibility of a satisfactory population prospect axiology: a satisfactory betterness ordering over alternatives that have some probability of bringing about one or more distinct populations. Since all of our population-affecting actions have a non-zero probability of bringing about

\footnotetext{
${ }^{1}$ See, for example, Parfit (1984, chap. 19), Ng (1989), Blackorby and Donaldson (1991), Carlson (1998), Kitcher (2000), and Tännsjö (2002).

2 The first four theorems are in Arrhenius (2000). The fifth is in (2003) and the sixth is in (2009; 2011). All six are collated in (forthcoming).

3 Thomas (2018) calls the assumption 'Small Steps'.
} 
more than one distinct population, it is population prospect axiologies that are of practical relevance, and these amended theorems state that no such axiology can satisfy each of a small number of compelling adequacy conditions. The key difference is that these theorems employ risky versions of Arrhenius's original conditions. The original conditions mandate, roughly, that a drop in welfare for one person can be compensated by a large enough increase in welfare elsewhere. The risky versions mandate, again roughly, that a slightly increased risk of a drop in welfare for one person can be compensated by a large enough increase in welfare elsewhere. These risky adequacy conditions are compelling even if Finite FineGrainedness is false, so lexical views do not escape these amended theorems.

I begin in Sect. 2 by outlining the framework of this paper more precisely. Then in Sect. 3 I formulate the adequacy conditions for Arrhenius's favoured Sixth Impossibility Theorem. I give some prima facie reasons to doubt Finite FineGrainedness in Sect. 4, after which I sketch out a simple lexical view and explain how it escapes the Sixth Theorem. Then in Sect. 5 I present a risky version of the theorem that does not depend on the truth of Finite Fine-Grainedness. I prove that Arrhenius's other impossibility theorems can be patched up with a similar manoeuvre in the Appendix.

\section{The framework}

In this paper, I use definitions and structural assumptions broadly in line with those of Arrhenius (2011; forthcoming). Two exceptions are worth noting. First, I borrow notation from Thomas's manuscript ${ }^{4}$ to simplify the presentation of the adequacy conditions and proofs. Second, I drop the assumption of Finite Fine-Grainedness in Sect. 5 and substitute new assumptions about the ordering of population prospects.

Arrhenius's impossibility theorems make extensive use of the notion of welfare: a measure of how good a person's life is for them. Lives are individuated by the person whose life it is and the kind of life it is, and it is assumed that the "has at least as high welfare as' relation applied to the set of possible lives is reflexive and transitive, but not necessarily complete. Life $x$ is better than life $y$ iff $x$ has at least as high welfare as $y$ and $y$ does not have at least as high welfare as $x$. Lives $x$ and $y$ are incommensurable iff $x$ does not have at least as high welfare as $y$ and $y$ does not have at least as high welfare as $x .^{5}$ Lives $x$ and $y$ are equally good iff $x$ has at least as high welfare as $y$ and $y$ has at least as high welfare as $x$. If two lives are equally good, they are at the same welfare level.

A life is neutral iff it is equally good for the person living it as some standard. This standard is defined differently by different authors. Arrhenius $(2011,5)$ defines it as a neutral welfare component: a component that makes a person's life neither better nor worse. Others define it as nonexistence (Arrhenius \& Rabinowicz, 2015)

\footnotetext{
${ }^{4}$ http://users.ox.ac.uk/ mert2060/webfiles/Reconstructing-Arrhenius-for-web.pdf.

5 We might instead claim that $x$ and $y$ are on a par or imprecisely equally good in this case. For the purposes of this the paper, the distinction between these relations is unimportant. See Chang (2016) for discussion.
} 
or a life constantly at a neutral level of temporal welfare (Broome, 2004, 68; Bykvist, 2007, 101). My discussion is compatible with all such definitions. A life is at a positive welfare level iff it is better than the standard, and at a negative welfare level iff it is worse than the standard.

Arrhenius assumes Finite Fine-Grainedness:

\section{Finite Fine-Grainedness}

There exists a finite sequence of slight welfare differences between any two welfare levels. (Arrhenius, 2016, 171; forthcoming)

We can leave 'slight' to be understood intuitively for now. Suppose, for example, that $x$ is a long life and $y$ is an otherwise identical life featuring one less second of mild pleasure. The difference between the welfare levels of $x$ and $y$ would certainly qualify as slight.

Arrhenius uses Finite Fine-Grainedness to ensure the existence of a finite, linearly ordered set of welfare levels, $\mathbb{W}$, with two properties:

1. The set ranges from a very negative welfare level, through a barely negative welfare level and three barely positive welfare levels, each higher than the last, up to three very positive welfare levels, each higher than the last.

2. The difference between adjacent welfare levels is slight.

We can represent the welfare levels in $\mathbb{W}$ with integers ranging from $\omega$ up to $\beta+2$ :

$$
\omega<\ldots<-1<0<1<2<3<\ldots<\beta<\beta+1<\beta+2
$$

Here 0 represents the neutral welfare level, -1 represents a barely negative level, and 1,2 , and 3 represent barely positive levels. $\omega$ represents a very negative level, and $\beta$ and above represent very positive levels. These are all the welfare levels employed in Arrhenius's proofs.

A population is a set of lives in a possible world. A population axiology is an 'at least as good as' relation on the set of all possible populations: reflexive and transitive, but not necessarily complete. Population $X$ is better than population $Y$ iff $X$ is at least as good as $Y$ and $Y$ is not at least as good as $X$. Populations $X$ and $Y$ are incommensurable iff $X$ is not at least as good as $Y$ and $Y$ is not at least good as $X{ }^{6}$ Population $X$ is equally good as population $Y$ iff $X$ is at least as good as $Y$ and $Y$ is at least as good as $X$. If two populations are equally good, they have the same value.

Two features of Arrhenius's adequacy conditions are worth noting. The first is that they quantify over $\mathbb{W}$. This set may be a proper subset of the set of all welfare levels, but that possibility is of little consequence. If no population axiology can satisfy Arrhenius's adequacy conditions quantifying over $\mathbb{W}$, then no population axiology can satisfy those adequacy conditions quantifying over all welfare levels. The second is that each adequacy condition includes an 'other things being equal' clause. That is needed because populations determine facts besides the distribution of welfare, and these facts might be axiologically relevant. The purpose of the 'other things being equal' clause is to hold all such non-welfare facts fixed.

\footnotetext{
$\overline{{ }^{6} \text { Or else they are on a par or imprecisely equally good. See footnote } 5 .}$
} 
In what follows, I use $\llbracket a \rrbracket$ to denote a population of one life at welfare level $a$, and $m \llbracket a \rrbracket$ to denote a population of $m$ lives at $a$. Uppercase letters like $A, B, X$, and $Y$ denote populations which may feature lives at more than one welfare level. Populations represented by different letters should be understood as pairwise disjoint so that, for example, $X$ and $m \llbracket a \rrbracket$ have no lives in common. $X+m \llbracket a \rrbracket$ then denotes a population of all the lives in $X$ and all the lives in $m \llbracket a \rrbracket$. I leave the 'other things being equal' clause in each adequacy condition implicit. With that proviso, $\succ$ denotes 'is better than' and $\succeq$ denotes 'is at least as good as.'

\section{Arrhenius's Sixth Impossibility Theorem}

Arrhenius's favoured Sixth Impossibility Theorem employs the following five adequacy conditions:

Egalitarian Dominance: If population $A$ is a perfectly equal population of the same size as population $B$, and every person in $A$ has higher welfare than every person in $B$, then $A$ is better than $B$.

Egalitarian Dominance (exact formulation): For any $a \in \mathbb{W}$ any $n \in \mathbb{N}$, and any population $X$ of size $n$ with all lives at welfare levels below $a$

$$
X \prec n \llbracket a \rrbracket
$$

General Non-Extreme Priority: For any welfare level $a$, there exists a number $n$ of lives such that, for any population $X$, a population consisting of $X, n$ very positive welfare lives, and one life at welfare level $a$ is at least as good as a population consisting of $X, n$ barely positive welfare lives, and one life at a welfare level slightly above $a$.

General Non-Extreme Priority (exact formulation): For any $a \in \mathbb{W}$, there exists $n \in \mathbb{N}$ such that, for any $b, c \in \mathbb{W}$ with $0<b \leq 3, c \geq \beta$, and any population $X$,

$$
X+\llbracket a+1 \rrbracket+n \llbracket b \rrbracket \preceq X+\llbracket a \rrbracket+n \llbracket c \rrbracket
$$

Non-Elitism: For any welfare levels $a, b$, and $c, a$ slightly higher than $b$ and $b$ higher than $c$, and for any one-life population $A$ at welfare level $a$, there is a population $C$ at welfare level $c$, and a population $B$ of the same size as $A+C$ such that, for any population $X$ consisting of lives with welfare ranging from $c$ to $a$, $X+B$ is at least as good as $X+A+C$.

Non-Elitism (exact formulation): For any $a, c \in \mathbb{W}$ with $a-1>c$, there exists $n \in \mathbb{N}$ such that, for any population $X$ with welfare levels ranging from $c$ to $a$,

$$
X+\llbracket a \rrbracket+n \llbracket c \rrbracket \preceq X+\llbracket a-1 \rrbracket+n \llbracket a-1 \rrbracket
$$

Weak Non-Sadism: There is a negative welfare level and a number of lives at this level such that the addition of any number of lives with positive welfare is at least as good as the addition of the lives with negative welfare.

Weak Non-Sadism (exact formulation): There exists $a \in \mathbb{W}$ with $a<0$ and $m \in \mathbb{N}$ such that, for any welfare level $b \in \mathbb{W}$ with $b>0$, any $n \in \mathbb{N}$, and any population $X$, 


$$
X+m \llbracket a \rrbracket \preceq X+n \llbracket b \rrbracket
$$

Weak Quality Addition: There is a number of very negative welfare lives such that, for any population $X$, there is a number of very positive welfare lives such that the addition of the very positive welfare lives to $X$ is at least as good as the addition of the very negative welfare lives plus any number of barely positive welfare lives to $X$.

Weak Quality Addition (exact formulation): There exists $a \in \mathbb{W}$ with $a<0$ and $m \in \mathbb{N}$ such that, for any population $X$, there exists $b, c \in \mathbb{W}$ with $0<b \leq 3$, $c \geq \beta$, and $n \in \mathbb{N}$, such that for any $q \in \mathbb{N}^{7}$

$$
X+m \llbracket a \rrbracket+q \llbracket b \rrbracket \preceq X+n \llbracket c \rrbracket
$$

Arrhenius's Sixth Impossibility Theorem states that these five adequacy conditions are incompatible:

\section{Arrhenius's Sixth Impossibility Theorem}

There is no population axiology which satisfies Egalitarian Dominance, General Non-Extreme Priority, Non-Elitism, Weak Non-Sadism, and Weak Quality Addition. (Arrhenius, 2011, 9; forthcoming)

However, the theorem is only true given Finite Fine-Grainedness. I prove that this is so in the next section, by presenting Lexical Totalism as a counterexample to the theorem. But the rough idea is as follows. Arrhenius assumes that, while single applications of Non-Elitism and General Non-Extreme Priority reduce a person's welfare only slightly, repeated applications of these conditions can reduce a very positive welfare level to a very negative welfare level. As we will see, this assumption is exactly what Lexical Totalism denies.

\section{Lexical Totalism}

Recall Finite Fine-Grainedness:

\section{Finite Fine-Grainedness}

There exists a finite sequence of slight welfare differences between any two welfare levels. (Arrhenius, 2016, 171; forthcoming)

Although this assumption might seem compelling, there are prima facie reasons to doubt it. Consider the following case from Roger Crisp:

\footnotetext{
7 This condition differs slightly from that of Arrhenius (2011). Arrhenius has the first two quantifiers the other way around, so that the condition begins 'For any population $X$, there is...' $(2011,9)$. As Thomas's manuscript notes, the Sixth Impossibility Theorem actually requires the slightly stronger condition stated here. In any case, the stronger version remains a compelling adequacy condition.
} 


\section{Haydn and the Oyster}

You are a soul in heaven waiting to be allocated a life on Earth. It is late Friday afternoon, and you watch anxiously as the supply of available lives dwindles. When your turn comes, the angel in charge offers you a choice between two lives, that of the composer Joseph Haydn and that of an oyster. Besides composing some wonderful music and influencing the evolution of the symphony, Haydn will meet with success and honour in his own lifetime, be cheerful and popular, travel, and gain much enjoyment from field sports. The oyster's life is far less exciting. Though this is rather a sophisticated oyster, its life will consist only of mild sensual pleasure, rather like that experienced by humans when floating very drunk in a warm bath. When you request the life of Haydn, the angel sighs, 'I'll never get rid of this oyster life. It's been hanging around for ages. Look, I'll offer you a special deal. Haydn will die at the age of seventy-seven. But I'll make the oyster life as long as you like.' (Crisp, 1997, 24; 2006, 112; see also McTaggart, 1927, 452-53)

Suppose that, as the oyster, you would never get bored of your mild sensual pleasure. Many of us share the following two intuitions about this case:

1. Increasing the length of the oyster life by one day increases its welfare level by some slight but constant amount.

2. An oyster life of any length is at a lower welfare level than the life of Haydn.

This combination of intuitions casts doubt on Finite Fine-Grainedness, for although each added day of oyster life yields a constant increase in welfare level, no number of additional days can make the oyster life at least as good as the life of Haydn. ${ }^{8}$ What's more, we might think that the only improvements that could bring the oyster life up to Haydn's welfare level do not come in slight increments. Suppose, for example, that the oyster life could be at least as good as Haydn's only if we endowed the oyster with autonomy, or made it capable of love, or gave its life meaning. Suppose further that no lives differing in their quantities of autonomy, love, or meaning differ only slightly in welfare. In that case, Finite Fine-Grainedness would be false.

We might try to account for these intuitions by claiming that the life of Haydn is of infinite value relative to the oyster life, but there are good reasons to avoid this move. One is that, if the value of Haydn's life is infinite, then the expected value of any prospect with a non-zero probability of resulting in Haydn's life is also infinite. A prospect that results in Haydn's life for certain has the same infinite expected value as a prospect that results in Haydn's life with probability one-in-a-hundred and an oyster life otherwise.

A better way of accounting for these intuitions is to represent welfare levels with a vector. For example, we can have the welfare level of a life $x$ as a vector of higher

\footnotetext{
8 The truth of these intuitions would not themselves contradict Finite Fine-Grainedness, because it could be that some other way of slightly increasing the oyster's welfare could eventually render the oyster life at least as good as the life of Haydn. However, as Carlson (forthcoming) points out, their truth would contradict Finite Fine-Grainedness if we also assume that a difference in welfare levels is slight only if it is not infinitely greater than some other difference in welfare levels.
} 
and lower goods - $\left(h_{x}, l_{x}\right)$ - each representable by integers without upper or lower bound. These welfare levels can then be ordered lexically, so that a welfare level $\left(h_{x}, l_{x}\right)$ is at least as high as a welfare level $\left(h_{y}, l_{y}\right)$ iff either $h_{x}>h_{y}$ or $h_{x}=h_{y}$ and $l_{x} \geq l_{y}$. We can specify that autonomy, love, and meaning are higher goods, while sensual pleasure is a lower good. Given this specification, the life of Haydn contains some non-zero quantity of higher goods and the oyster life contains none. The lexical ordering can then account for both of our intuitions. Increasing the length of the oyster life by one day increases its quantity of lower goods by some slight but constant amount, but no quantity of lower goods in an oyster life can match the non-zero quantity of higher goods in the life of Haydn. And extending this lexical ordering to cover prospects gives the right results in the risky case outlined above. Let Haydn's welfare level be $(a, 0)$ with $a>0$ and the oyster's welfare level $(0, b)$ with $b>0$, and define the expected value of a prospect as a probability-weighted average of the values of its possible outcomes. Then the expected value of the prospect that results in Haydn's life for certain is $(a, 0)$ and the expected value of the prospect that results in Haydn's life with probability one-in-ahundred and an oyster life otherwise is $(0.01 a, 0.99 \mathrm{~b})$. Since $a>0.01 a$, the lexical ordering has the former prospect better than the latter.

We can follow Carlson (forthcoming) in filling out the view as follows:

A welfare level $\left(h_{x}, l_{x}\right)$ is

positive iff $h_{x}>0$, or $h_{x}=0$ and $l_{x}>0$,

negative iff $h_{x}<0$, or $h_{x}=0$ and $l_{x}<0$,

neutral iff $h_{x}=0$ and $l_{x}=0$,

very positive iff $h_{x} \geq e$, for a particular positive integer $e$,

barely positive only if $h_{x}=0$ and $l_{x}>0$,

barely negative only if $h_{x}=0$ and $l_{x}<0$, and

very negative iff $h_{x} \leq f$, for a particular negative integer $f$.

A welfare level $\left(h_{x}, l_{x}\right)$ is merely slightly higher than a welfare level $\left(h_{y}, l_{y}\right)$ only if $h_{x}=h_{y}$ and $l_{x}=l_{y}+u, u>0$.

On this view, Finite Fine-Grainedness is false. A welfare difference is slight only if it involves no change in the quantity of higher goods, so no number of slight welfare differences can bridge the gap between welfare levels that differ in their quantity of higher goods.

We can order populations in the same way that we order lives. Let the value of a population $X$ be represented by the vector $\left(h_{X}, l_{X}\right)$, where $h_{X}$ is the sum of all the higher goods and $l_{X}$ is the sum of all the lower goods in the lives in $X$. Population $X$ is at least as good as population $Y$ iff either $h_{X}>h_{Y}$ or $h_{X}=h_{Y}$ and $l_{X} \geq l_{Y}$. Call this population axiology Lexical Totalism.

As Thomas (2018) and Carlson (forthcoming) note, Lexical Totalism is a counterexample to Arrhenius's Sixth Impossibility Theorem. It satisfies all five adequacy conditions. It satisfies Egalitarian Dominance because every person in $A$ having higher welfare than every person in $B$ entails that total welfare in $A$ is higher than in $B$. And it satisfies Weak Non-Sadism because adding any number of negative welfare lives reduces total welfare while adding any number of positive 
welfare lives increases it. Weak Quality Addition is satisfied for a similar reason. Adding any number of very positive welfare lives increases total welfare, while adding any combination of very negative welfare lives and barely positive welfare lives reduces it. More precisely, let $\left(h_{X}, l_{X}\right)$ represent the value of $X$. Adding very positive welfare lives means adding $(p, q)$ with $p>0$, while adding a combination of very negative welfare lives and barely positive welfare lives means adding $(r$, $s)$ with $r<0$. Weak Quality Addition is satisfied because $\left(h_{X}+p, l_{X}+q\right)$ is greater than $\left(h_{X}+r, l_{X}+s\right)$ no matter what values $q$ and $s$ take.

Lexical Totalism also satisfies General Non-Extreme Priority. Let $\left(h_{a}, l_{a}\right)$ represent welfare level $a$. Then a population consisting of $X$, one life at $a$, and some number of very positive welfare lives has a value of $\left(h_{X}+h_{a}+p, l_{X}+l_{a}+q\right)$ with $p>0$. Meanwhile, a population consisting of $X$, one life at a welfare level slightly above $a$ (represented by $\left(h_{a}, l_{a}+u\right)$ with $\left.u>0\right)$, and some number of barely positive welfare lives has a value of $\left(h_{X}+h_{a}, l_{X}+l_{a}+u+s\right)$ with $u>0, s>0$. General Non-Extreme Priority is satisfied because $\left(h_{X}+h_{a}+p, l_{X}+l_{a}+q\right)$ is greater than $\left(h_{X}+h_{a}, l_{X}+l_{a}+u+s\right)$ no matter what values $q, u$, and $s$ take.

Non-Elitism completes the set. Again, let $\left(h_{a}, l_{a}\right)$ represent welfare level $a$, so that $\left(h_{a}\right.$, $l_{a}-u$ ) with $u>0$ represents welfare level $b$, and let $\left(h_{c}, l_{c}\right)$ represent welfare level $c$. Then the value of $X+B$ is $\left(h_{X}+n\left(h_{a}\right), l_{X}+n\left(l_{a}-u\right)\right)$ and the value of $X+A+C$ is $\left(h_{X}+h_{a}+(n-1)\left(h_{c}\right), l_{X}+l_{a}+(n-1)\left(l_{c}\right)\right)$. Cancelling the $h_{X}$ and $l_{X}$ terms, we can see that Non-Elitism is satisfied: $\left(n\left(h_{a}\right), n\left(l_{a}-u\right)\right)$ is at least as good as $\left(h_{a}+(n-1)\left(h_{c}\right), l_{a}+(n-1)\left(l_{c}\right)\right)$ for some $n \in \mathbb{N}$, since $h_{a}>h_{c}$ or $h_{a}=h_{c}$ and $\left(l_{a}-u\right)>l_{c}$.

Therefore, Arrhenius's Sixth Impossibility Theorem is escapable. Population axiologies that deny Finite Fine-Grainedness can satisfy all of its adequacy conditions. What's more, these axiologies have other advantages besides. Lexical Totalism coheres nicely with our intuitions in cases like Haydn and the Oyster, its lexical ordering of lives admits of a natural extension to populations and prospects, and all the while it remains faithful to the appealing idea that a population is at least as good as another iff it contains at least as much welfare.

Lexical Totalism also satisfies all the adequacy conditions in Arrhenius's First, Fourth, and Fifth Impossibility Theorems. ${ }^{9}$ The Second and Third Impossibility Theorems are a different matter. They feature the following adequacy condition:

Inequality Aversion: For any welfare levels $a, b$, and $c, a$ higher than $b$, and $b$ higher than $c$, and for any population $A$ with welfare $a$, there is a larger population $C$ with welfare $c$, such that a perfectly equal population $B$ of the same size as $A+C$ and with welfare $b$, is at least as good as $A+C$.

Lexical Totalism violates this condition when, for example, $a$ is a very positive welfare level and $b$ and $c$ are barely positive welfare levels. However, in this case, Inequality Aversion does not seem particularly compelling. Suppose, for example, that $a$ is the welfare level enjoyed by Haydn, $b$ is the welfare level enjoyed by an oyster that lives one-hundred years, and $c$ is the welfare level enjoyed by an oyster

9 As Carlson (forthcoming) proves. 
that lives ninety-nine years. Inequality Aversion states that, for any number $m$ of lives equally good as Haydn's, there is some number $n$ of ninety-nine year oyster lives such that $m+n$ one-hundred year oyster lives are at least as good as $m$ Haydnquality lives and $n$ ninety-nine year oyster lives.

In fact, Arrhenius acknowledges that Inequality Aversion is not particularly compelling considered alone (forthcoming, 147). But he defends it by deriving it from the more compelling Non-Elitism condition (forthcoming, 150f.). His derivation, however, depends on Finite Fine-Grainedness (forthcoming, 323-26). If we deny Finite Fine-Grainedness, no such thing follows. Therefore, advocates of Lexical Totalism can claim that their view satisfies all of the compelling adequacy conditions in each of Arrhenius's six impossibility theorems.

Kitcher (2000), Thomas (2018), Nebel (2021), and Carlson (forthcoming) offer lexical views along these lines. As they note, these views can be tweaked and generalised in various ways. Welfare levels could be represented by vectors with any number of elements, each element could be represented by any subset of the real numbers, and the ordering could employ thresholds of various kinds to account for incommensurability. Suppose, for example, that population $X$ is at least as good as population $Y$ iff either $h_{X}-h_{Y}>\Delta$ or $h_{X} \geq h_{Y}$ and $l_{X} \geq l_{Y}$. In that case, it could be that neither of $X$ and $Y$ is at least as good as the other. It could also be indeterminate whether the quantity of higher goods in a life exceeds some threshold, in which case the ordering of lives and populations will also admit of indeterminacy.

All such views, however, must deny Finite Fine-Grainedness to avoid Arrhenius's Sixth Impossibility Theorem, and we might complain that this denial is not well-motivated. ${ }^{10}$ One line of argument in favour of Finite Fine-Grainedness is as follows. Every plausible candidate for being a higher good (e.g. autonomy, love, meaning) comes in fine-grained quantities, and if two lives are identical but for a slight difference in their quantity of some higher good, they differ only slightly in welfare. These two premises imply Finite Fine-Grainedness.

This argument has some force, but it is hardly irresistible. Deniers of Finite FineGrainedness point out that the nature of welfare remains an open question (Thomas, 2018, 829-30; Nebel, 2021, 10, 36; Carlson, forthcoming, 22-26). We simply do not know what makes a life good, and so we do not know that higher goods are fine-grained. What's more, they can draw on a whole array of axiological phenomena to flesh out the case for doubt. Mill's distinction between higher and lower pleasures is one starting point. He claims that some pairs of pleasures are such that 'those who are competently acquainted with both' place one 'so far above the other that they... would not resign it for any quantity of the other pleasure.' (Mill, 1861, chap. 2, para. 5). And there is no smooth sequence between these higher and lower pleasures because they depend on different faculties. Higher pleasures depend on our 'intellect,' 'imagination,' and 'moral sentiments,' while lower pleasures require only 'mere sensation.' (Mill, 1861, chap. 2, para. 4). From this foundation, it is just a short step to the claim that a life featuring higher pleasures differs markedly in welfare from any life lacking them.

\footnotetext{
${ }^{10}$ Another objection is that lexical views imply the lexical dilemma. See Thornley (forthcoming) for this objection and a response.
} 
Another argument comes from Nebel. He suggests that even if autonomy and meaning are fine-grained, the primary determinant of welfare might be the binary instantiation of these goods (Nebel, 2021, 11-12). Perhaps no life that is meaningful simpliciter is merely slightly better than a life that is meaningless simpliciter. Granted, 'meaningful' is almost certainly a vague term, but that is no reason to reject the view. Many compelling moral principles contain vague terms. One example is the claim that it is wrong to experiment on a subject that has not given their informed consent. And vagueness plays a key role in many population axiologies too. Broome (2004, 180-82), for example, claims that it can be vague whether a life is better lived than not lived.

Meanwhile, Griffin $(1988,86)$ and Carlson (forthcoming) suggest that higher goods might be a composite of other goods, none of which is in itself higher. A life might have to instantiate autonomy, love, knowledge, virtue, and meaning to some degree in order to reach a very positive welfare level, and any life instantiating just four of these five goods might be at a welfare level markedly lower. The presence of all five might be a kind of Moorean 'organic unity' in which the whole is more than the sum of its parts (Moore, 1903, 78-80).

These accounts are incomplete, but plausible enough in their outlines. Therefore, we cannot conclude that a population axiology is unsatisfactory simply because it violates Finite Fine-Grainedness, and any argument to this effect must reckon with a whole array of axiological phenomena. Determining whether a satisfactory population axiology is possible thus seems to require resolving some tricky questions about the nature of welfare.

I claim, however, that no such axiological enquiries are necessary. What matters for all practical purposes is the possibility of a satisfactory population prospect axiology, and the impossibility of such an axiology can be proved without employing Finite Fine-Grainedness. The key insight is that expected welfare levels are finitely fine-grained, even if welfare levels are not.

\section{The Risky Sixth Impossibility Theorem}

My risky versions of Arrhenius's impossibility theorems employ the notion of a population prospect which I define, somewhat clunkily, as an alternative with some non-zero probability of bringing about one or more distinct populations. These population prospects can be divided into the trivial and the non-trivial. Trivial population prospects are those alternatives that bring about some population with probability 1. Non-trivial population prospects are those alternatives that bring about two or more distinct populations with probabilities strictly between 0 and $1 .{ }^{11}$

\footnotetext{
11 These definitions are in line with those given by Arrhenius and Stefánsson (2020) in their manuscript on population ethics under risk. Arrhenius and Stefánsson also offer impossibility theorems in population prospect axiology. However, their theorems employ different axioms to the theorems below. Their axioms do not so obviously dispense with the need to assume Finite Fine-Grainedness.

As Arrhenius and Stefánsson note, the literature in population ethics has thus far mostly disregarded questions of risk. For exceptions, see Blackorby, Bossert, and Donaldson (2005), Roberts (2007), Asheim
} 
We might denote non-trivial population prospects with $\left[p_{1} X_{1}, \ldots, p_{n} X_{n}\right]$, where each $p_{i}$ is a probability (with $0<p_{i}<1$ and $p_{1}+\cdots+p_{n}=1$ ) and each $X_{i}$ is a population. The prospect $\left[p_{1} X_{1}, \ldots, p_{n} X_{n}\right]$ brings about $X_{1}$ with probability $p_{1}, X_{2}$ with probability $p_{2}$, and so on. However, this notation quickly becomes unwieldy for prospects that bring about different sets of lives with different probabilities. Suppose, for example, that a prospect brings about $\llbracket a \rrbracket$ with probability $p, \llbracket a-1 \rrbracket$ with probability $1-p, Y$ with probability 1 , and no other lives with non-zero probability. We could denote this prospect with $[(p)(\llbracket a \rrbracket+Y),(1-p)(\llbracket a-1 \rrbracket$ $+Y)]$, but it is simpler to separate those populations brought about with probability less than 1 from those populations brought about with certainty, so that we denote the prospect with $[(p) \llbracket a \rrbracket,(1-p) \llbracket a-1 \rrbracket]+Y$. I adopt the simpler convention in what follows.

Given my definitions, Arrhenius's original theorems can be understood as stating that there is no satisfactory betterness ordering over trivial population prospects. I go beyond these theorems in assuming that the 'at least as good as' relation applies to non-trivial population prospects as well as trivial ones. More precisely, I assume that the 'at least as good as' relation is reflexive over the set of population prospects and that it holds, at least sometimes, when one or both of its relata are non-trivial population prospects. This assumption seems difficult to deny. Suppose that a first alternative brings about a population of one million people living wonderful lives with probability 0.5 and a population of one million people living almost-wonderful lives otherwise, and that a second alternative brings about a population of one million people living awful lives with probability 1 . It seems obvious that the first alternative is better than the second. ${ }^{12}$

What's more, denying that any non-trivial population prospect is better than any other would strip one's chosen population axiology of all practical relevance, since all of our population-affecting actions have some non-zero probability of bringing about more than one distinct population. Suppose, for example, that a government minister is considering a policy that would reduce the cost of childcare. Whether she implements the policy or not, there is no single population that will come about with probability 1 , so all of her alternatives are non-trivial population prospects. If no such prospects are better than any others, then population axiology cannot inform her decision. The same goes for more personal decisions. My having a child has a non-zero probability of resulting in more than one distinct population, because it is uncertain how many children my child will have. And the effects of refraining are not certain either. There is always a chance that it will spur a government minister to implement a policy reducing the cost of childcare.

\section{Footnote 11 continued}

and Zuber (2016), Thomas (2016), Nebel (2017, 2019, 2021), Budolfson and Spears (2018), and Spears and Budolfson (2019).

12 In this example, the first alternative stochastically dominates the second. But there are other compelling examples of betterness over prospects that do not have this feature. Suppose, for example, that a first alternative brings about a population of one million people living wonderful lives with probability 1 , and a second alternative brings about a population of one million people living ever-so-slightly-betterthan-wonderful lives with probability 0.00001 and a population of one million people living awful lives otherwise. The first alternative seems better than the second. 
I also assume that the 'at least as good as' relation is transitive over the set of population prospects. Some authors deny the transitivity assumption in Arrhenius's original impossibility theorems (Rachels, 2004; Temkin, 2012), and one might be tempted to do the same here. However, this move strikes most as a drastic step. At worst, it is denying a logical truth (Broome, 2004, chap. 4). At best, it requires a radical upheaval of axiology and practical rationality.

Recall that Arrhenius uses Finite Fine-Grainedness to ensure the existence of a finite, linearly ordered set of welfare levels, $\mathbb{W}$, with two properties:

1. The set ranges from a very negative welfare level, through a barely negative welfare level and three barely positive welfare levels, each higher than the last, up to three very positive welfare levels, each higher than the last.

2. The difference between adjacent welfare levels is slight.

If Finite Fine-Grainedness is false in the way that Lexical Totalists suggest, there is no such set. But there will still be finite, linearly ordered sets of welfare levels with just the first property. We can pick out one such set in which many of the differences between adjacent welfare levels are slight, and those differences that are not slight are not egregiously big either. Call this set $W^{*}$. As before, we can represent these welfare levels with integers ranging from $\omega$ up to $\beta+2$ :

$$
\omega<\ldots<-1<0<1<2<3<\ldots<\beta<\beta+1<\beta+2
$$

Again, 0 represents the neutral welfare level, -1 represents a barely negative level, 1,2 , and 3 represent barely positive levels, $\omega$ represents a very negative level, and $\beta$ and above represent very positive levels. This time, however, there is at least one pair of adjacent welfare levels that differ more than slightly.

Two features that my adequacy conditions share with Arrhenius's are worth reiterating. First, my adequacy conditions quantify over $\mathbb{W} *$. Like Arrhenius's $\mathbb{W}$, this set may be a proper subset of all possible welfare levels, but that possibility is of little consequence. If no population prospect axiology can satisfy these adequacy conditions quantifying over $\mathbb{W}^{*}$, then no population prospect axiology can satisfy these adequacy conditions quantifying over all welfare levels. The second is that my adequacy conditions also leave an 'other things being equal' clause implicit.

Now recall the General Non-Extreme Priority and Non-Elitism conditions employed in Arrhenius's Sixth Impossibility Theorem. Applied to $\mathbb{W}^{*}$, the informal statements and the exact formulations of these conditions come apart. The informal statements refer to welfare levels that differ slightly while the exact formulations refer to adjacent welfare levels, and $\mathbb{W} *$ features at least one pair of adjacent welfare levels that differ more than slightly. As we have seen, the informal versions of Arrhenius's adequacy conditions are not incompatible over $\mathbb{W} *$, because repeated applications of these conditions cannot reduce a very positive welfare level to a very negative one. They cannot 'jump the gap' between the pair(s) of adjacent welfare levels that differ more than slightly. The exact formulations, on the other hand, are incompatible over $\mathbb{W}^{*}$, since they pay no heed to the size of the difference between adjacent welfare levels. Applied to $\mathbb{W}^{*}$, the conditions are as follows: 
General Non-Extreme Priority over $\mathbb{W}^{*}$ (exact formulation): For any $a \in \mathbb{W}^{*}$, there exists $n \in \mathbb{N}$ such that, for any $b, c \in \mathbb{W}^{*}$ with $0<b \leq 3, c \geq \beta$, and any population $X$,

$$
X+\llbracket a+1 \rrbracket+n \llbracket b \rrbracket \preceq X+\llbracket a \rrbracket+n \llbracket c \rrbracket
$$

Non-Elitism over $\mathbb{W} *$ (exact formulation): For any $a, c \in \mathbb{W} *$ with $a-1>c$, there exists $n \in \mathbb{N}$ such that, for any population $X$ with welfare levels ranging from $c$ to $a$,

$$
X+\llbracket a \rrbracket+n \llbracket c \rrbracket \preceq X+\llbracket a-1 \rrbracket+n \llbracket a-1 \rrbracket
$$

However, both of these conditions are open to doubt. Consider first General NonExtreme Priority. Suppose that the difference between welfare levels $a$ and $a+1$ is not slight. Perhaps $a+1$ corresponds to a life featuring only 'lower bads' like nondebilitating harm, whereas $a$ corresponds to a life featuring 'higher bads' like debilitating harm (see Handfield \& Rabinowicz, 2018). In that case, we might claim that $X+\llbracket a \rrbracket+n \llbracket c \rrbracket$ is worse than $X+\llbracket a+1 \rrbracket+n \llbracket b \rrbracket$ no matter how large $n$ is. No number of very positive welfare lives can make up for a life featuring debilitating harm.

We might doubt Non-Elitism for a similar reason. Suppose this time that the difference between welfare levels $a$ and $a-1$ is not slight. Perhaps $a$ corresponds to a life featuring a higher good like autonomy, whereas $a-1$ and $c$ correspond to lives featuring only lower goods like sensual pleasure. In that case, we might claim that $X+\llbracket a-1 \rrbracket+n \llbracket a-1 \rrbracket$ is worse than $X+\llbracket a \rrbracket+n \llbracket c \rrbracket$ no matter how large $n$ is. No increase in the quantity of sensual pleasure can make up for the loss of autonomy.

However, I claim that the following risky versions of General Non-Extreme Priority and Non-Elitism are compelling, even quantified over $\mathbb{W} *$ :

Risky General Non-Extreme Priority (exact formulation): For any $a \in \mathbb{W}^{*}$, there exists $m \in \mathbb{N}$ and $p$ of the form $\frac{1}{r}$ with $r \in \mathbb{N}$ such that, for any $k \in \mathbb{R}$ with $0 \leq k \leq 1-p$, any $b, c \in \mathbb{W}^{*}$ with $0<b \leq 3, c \geq \beta$, and any population $X$, $X+[(1-k) \llbracket a \rrbracket,(k) \llbracket a-1 \rrbracket]+m \llbracket b \rrbracket \preceq X+[(1-k-p) \llbracket a \rrbracket,(k+p) \llbracket a-1 \rrbracket]+m \llbracket c \rrbracket$

Risky Non-Elitism (exact formulation): For any $a, c \in \mathbb{W}^{*}$ with $a-1>c$, there exists $m \in \mathbb{N}$ and $p$ of the form $\frac{1}{r}$ with $r \in \mathbb{N}$ such that, for any $k \in \mathbb{R}$ with $0 \leq k$ $\leq 1-p$ and any population $X$ consisting of lives with welfare ranging from $c$ to $a$,

$$
\begin{aligned}
X+[(1-k) \llbracket a \rrbracket,(k) \llbracket a-1 \rrbracket]+m \llbracket c \rrbracket \preceq & X+[(1-k-p) \llbracket a \rrbracket,(k+p) \llbracket a-1 \rrbracket] \\
& +m \llbracket a-1 \rrbracket
\end{aligned}
$$

This assortment of quantifiers and variables is somewhat difficult to parse, but the rough idea is as follows. Arrhenius's original conditions mandate that some fixed drop in welfare for one person can always be compensated by a rise in welfare for some number of other people. The risky versions mandate only that some fixed increase in the risk of some drop in welfare for one person can always be compensated by a rise in welfare for some number of other people. The size of this fixed 
increase in risk could be very small. The only restriction is that multiplying it by some natural number gives an answer of 1 . And that makes these risky conditions compelling even in cases where the original conditions are not. Consider again the case that casts doubt on General Non-Extreme Priority: $a$ corresponds to a life featuring some debilitating harm, $a+1$ corresponds to a life featuring only nondebilitating harm, $b$ corresponds to a barely positive welfare life, and $c$ corresponds to a very positive welfare life. Risky General Non-Extreme Priority states only that some tiny increase in the risk of a drop in welfare from a life of non-debilitating harm to a life featuring some debilitating harm can be compensated by raising some number of lives from barely positive welfare levels to very positive welfare levels. This increase in risk could be $10^{-100}(0.0 \ldots 1$ with 99 zeroes between the decimalpoint and the 1). To get a grip on just how small this increase is, consider the following. Suppose you had a biased coin that came up heads with probability $10^{-100}$. Even if you had flipped this coin one million times per millisecond from the Big Bang up until now, your chance of seeing one or more heads would still be less than $10^{-73}(0.0 \ldots 1$ with 72 zeroes between the decimal-point and the 1$) .{ }^{13}$ One person's undergoing this (nigh-on non-existent) increase in risk can surely be compensated by raising some number of lives from barely positive to very positive welfare levels.

The same goes for Risky Non-Elitism. It is compelling even in cases where the original Non-Elitism condition is not. Again, let $a$ correspond to a life featuring some higher good like autonomy, $a-1$ correspond to a life featuring only sensual pleasure, and $c$ correspond to a life featuring slightly less sensual pleasure. Risky Non-Elitism states only that some tiny increase in the risk of a drop in welfare from a life of autonomy to a life of sensual pleasure can be compensated by some increase in the quantity of sensual pleasure elsewhere. Again, this increase in risk could be a nigh-on non-existent $10^{-100}$. That makes Risky Non-Elitism very difficult to deny.

These risky conditions, in conjunction with the transitivity of the 'at least as good as' relation over population prospects, imply that the original conditions are true over the welfare levels in W*. Risky General Non-Extreme Priority plus transitivity implies General Non-Extreme Priority proper, and Risky Non-Elitism plus transitivity implies Non-Elitism proper, as I prove below. First, General NonExtreme Priority:

Fix any $a$ as in General Non-Extreme Priority. From Risky General Non-Extreme Priority, we obtain corresponding $m, p$, and $r$. Let $n=r m$. Consider the following population with any $b$ and $X$ as in General Non-Extreme Priority:

$$
X+\llbracket a+1 \rrbracket+n \llbracket b \rrbracket
$$

Since $n=r m$, the above can be expressed as follows, with all $m_{i}=m$ :

\footnotetext{
13 Rounding up the time since the Big Bang to 14 billion years, there have been $4.415 \times 10^{20}$ milliseconds between then and now. Flipping the coin one million times per millisecond give $4.415 \times$ $10^{26}$ coin flips. Subtracting $10^{-100}$ from 1 and raising the answer to the power of $4.415 \times 10^{26}$ gives the probability of seeing 0 heads. Subtracting this probability from 1 gives $4.415 \times 10^{-74}$.
} 


$$
X+\llbracket a+1 \rrbracket+m_{1} \llbracket b \rrbracket+m_{2} \llbracket b \rrbracket+m_{3} \llbracket b \rrbracket+\cdots+m_{r} \llbracket b \rrbracket
$$

Applying Risky General Non-Extreme Priority yields the following, with any $c$ as in General Non-Extreme Priority:

$$
\preceq X+[(1-p) \llbracket a+1 \rrbracket,(p) \llbracket a \rrbracket]+m_{1} \llbracket c \rrbracket+m_{2} \llbracket b \rrbracket+m_{3} \llbracket b \rrbracket+\cdots+m_{r} \llbracket b \rrbracket
$$

Applying it again yields:

$$
\preceq X+[(1-2 p) \llbracket a+1 \rrbracket,(2 p) \llbracket a \rrbracket]+m_{1} \llbracket c \rrbracket+m_{2} \llbracket c \rrbracket+m_{3} \llbracket b \rrbracket+\cdots+m_{r} \llbracket b \rrbracket
$$

Applying it $r-2$ more times yields:

$$
\preceq X+[(1-r p) \llbracket a+1 \rrbracket,(r p) \llbracket a \rrbracket]+m_{1} \llbracket c \rrbracket+m_{2} \llbracket c \rrbracket+m_{3} \llbracket c \rrbracket+\cdots+m_{r} \llbracket c \rrbracket
$$

Since $r p=1$, the above simplifies to:

$$
X+\llbracket a \rrbracket+m_{1} \llbracket c \rrbracket+m_{2} \llbracket c \rrbracket+m_{3} \llbracket c \rrbracket+\cdots+m_{r} \llbracket c \rrbracket
$$

Since $n=r m$, the above simplifies to:

$$
X+\llbracket a \rrbracket+n \llbracket c \rrbracket
$$

And by the transitivity of the 'at least as good as' relation, we can conclude:

$$
X+\llbracket a+1 \rrbracket+n \llbracket b \rrbracket \preceq X+\llbracket a \rrbracket+n \llbracket c \rrbracket
$$

Which is General Non-Extreme Priority, as desired.

Second, Non-Elitism:

Fix any $a, c$ as in Non-Elitism. From Risky Non-Elitism, we obtain corresponding $m, p$, and $r$. Let $n=r m$. Consider the following population with any $X$ as in NonElitism:

$$
X+\llbracket a \rrbracket+n \llbracket c \rrbracket
$$

Since $n=r m$, the above can be expressed as follows, with all $m_{i}=m$ :

$$
X+\llbracket a \rrbracket+m_{1} \llbracket c \rrbracket+m_{2} \llbracket c \rrbracket+m_{3} \llbracket c \rrbracket+\cdots+m_{r} \llbracket c \rrbracket
$$

Applying Risky Non-Elitism yields:

$$
\preceq X+[(1-p) \llbracket a \rrbracket,(p) \llbracket a-1 \rrbracket]+m_{1} \llbracket a-1 \rrbracket+m_{2} \llbracket c \rrbracket+m_{3} \llbracket c \rrbracket+\cdots+m_{r} \llbracket c \rrbracket
$$

Applying it again yields:

$\preceq X+[(1-2 p) \llbracket a \rrbracket,(2 p) \llbracket a-1 \rrbracket]+m_{1} \llbracket a-1 \rrbracket+m_{2} \llbracket a-1 \rrbracket+m_{3} \llbracket c \rrbracket+\cdots+m_{r} \llbracket c \rrbracket$

Applying it $r-2$ more times yields:

$$
\begin{aligned}
\preceq & X+[(1-r p) \llbracket a \rrbracket,(r p) \llbracket a-1 \rrbracket]+m_{1} \llbracket a-1 \rrbracket+m_{2} \llbracket a-1 \rrbracket+m_{3} \llbracket a-1 \rrbracket+\cdots \\
& +m_{r} \llbracket a-1 \rrbracket
\end{aligned}
$$

Since $r p=1$, the above simplifies to:

$$
X+\llbracket a-1 \rrbracket+m_{1} \llbracket a-1 \rrbracket+m_{2} \llbracket a-1 \rrbracket+m_{3} \llbracket a-1 \rrbracket+\cdots+m_{r} \llbracket a-1 \rrbracket
$$

Since $n=r m$, the above simplifies to: 


$$
X+\llbracket a-1 \rrbracket+n \llbracket a-1 \rrbracket
$$

And by the transitivity of the 'at least as good as' relation, we can conclude:

$$
X+\llbracket a \rrbracket+n \llbracket c \rrbracket \preceq X+\llbracket a-1 \rrbracket+n \llbracket a-1 \rrbracket
$$

Which is Non-Elitism, as desired.

The impossibility theorem can then be proved using Arrhenius's original conditions understood as adequacy conditions on population prospects and quantified over $\mathbb{W}^{*}$. The proof is isomorphic to that given by Arrhenius (2011; forthcoming), so I will not repeat it here. ${ }^{14}$ The conclusion is as follows:

\section{The Risky Sixth Impossibility Theorem}

There is no population prospect axiology which satisfies Egalitarian Dominance, Risky General Non-Extreme Priority, Risky Non-Elitism, Weak NonSadism, and Weak Quality Addition.

Each of these adequacy conditions is compelling even if Finite Fine-Grainedness is false, so lexical views do not escape this impossibility theorem. They must violate Risky General Non-Extreme Priority or Risky Non-Elitism, or else take the drastic step of claiming that the 'at least as good as' relation is intransitive over population prospects. Therefore, the Risky Sixth Impossibility Theorem demonstrates that there is no satisfactory population prospect axiology.

Acknowledgements I thank Teruji Thomas, William MacAskill, Andreas Mogensen, and an anonymous reviewer for helpful comments and discussion. This work was supported by an Arts and Humanities Research Council studentship.

Open Access This article is licensed under a Creative Commons Attribution 4.0 International License, which permits use, sharing, adaptation, distribution and reproduction in any medium or format, as long as you give appropriate credit to the original author(s) and the source, provide a link to the Creative Commons licence, and indicate if changes were made. The images or other third party material in this article are included in the article's Creative Commons licence, unless indicated otherwise in a credit line to the material. If material is not included in the article's Creative Commons licence and your intended use is not permitted by statutory regulation or exceeds the permitted use, you will need to obtain permission directly from the copyright holder. To view a copy of this licence, visit http:// creativecommons.org/licenses/by/4.0/.

\section{Appendix}

In this section, I prove that Arrhenius's other impossibility theorems can be patched up with a similar manoeuvre. Each can be turned into a theorem stating that no population prospect axiology satisfies a small number of adequacy conditions, independently of Finite Fine-Grainedness.

\footnotetext{
14 Although note that Thomas's manuscript points out that the proof in Arrhenius (2011) contains a minor mistake. The theorem actually requires the slightly stronger version of Weak Quality Addition formulated above. See footnote 7 .
} 


\section{The Risky First Impossibility Theorem}

Arrhenius's First Impossibility Theorem states that the following adequacy conditions are incompatible:

Egalitarian Dominance (exact formulation): For any $a \in \mathbb{W}$, any $n \in \mathbb{N}$, and any population $X$ of size $n$ with all lives at welfare levels below $a$,

$$
X \prec n \llbracket a \rrbracket
$$

Quantity (exact formulation): For any $a \in \mathbb{W}, a>1$, and $m \in \mathbb{N}$, there exists $n \in \mathbb{N}$ such that,

$$
m \llbracket a \rrbracket \preceq n \llbracket a-1 \rrbracket
$$

Quality (exact formulation): There exists $m \in \mathbb{N}$ such that, for any $n \in \mathbb{N}$,

$$
n \llbracket 3 \rrbracket \preceq m \llbracket \beta \rrbracket
$$

Quantity is not particularly compelling applied to $\mathbb{W}^{*}$, because some pair of welfare levels $a$ and $a-1$ differ more than slightly. However, Risky Quantity is compelling:

Risky Quantity (exact formulation): For any $a \in \mathbb{W}^{*}$ with $a>1$ and $m \in \mathbb{N}$, there exists $h \in \mathbb{N}$ and $p$ of the form $\frac{1}{r}$ with $r \in \mathbb{N}$ such that, for any $k \in \mathbb{R}$ with $0 \leq k \leq 1-p$ and $g \in \mathbb{N} \cup\{0\}$,

$$
\begin{gathered}
{[(1-k) m \llbracket a \rrbracket,(k) m \llbracket a-1 \rrbracket]+g \llbracket a-1 \rrbracket \preceq[} \\
+g \llbracket a-1 \rrbracket+h \llbracket a-1 \rrbracket \\
+g) m \llbracket a-1 \rrbracket]
\end{gathered}
$$

It states, roughly, that a fixed increase in the risk of a drop in welfare for the best-off in a population (from one positive welfare level to another) can always be compensated by the addition of some number of lives at the lower positive welfare level. Risky Quantity plus transitivity implies that Quantity is true over $\mathbb{W}^{*}$ :

Fix any $a$ and $m$ as in Quantity. From Risky Quantity, we obtain corresponding $p$ and $r$. We will apply Risky Quantity $r$ times, with different values of $g$. Consider first $g_{1}=0$. From Risky Quantity, we obtain $h_{1}$. Then inductively set $g_{i+1}=$ $g_{i}+h_{i}$ and obtain $h_{i+1}$. Finally, set $n=m+h_{1}+h_{2}+h_{3}+\cdots+h_{r}$. Consider the following population:

$$
m \llbracket a \rrbracket
$$

Applying Risky Quantity yields:

$$
\preceq[(1-p) m \llbracket a \rrbracket,(p) m \llbracket a-1 \rrbracket]+h_{1} \llbracket a-1 \rrbracket
$$

Applying it again yields: 


$$
\preceq[(1-2 p) m \llbracket a \rrbracket,(2 p) m \llbracket a-1 \rrbracket]+h_{1} \llbracket a-1 \rrbracket+h_{2} \llbracket a-1 \rrbracket
$$

Applying it $r-2$ more times yields:

$$
\preceq\left[(1-r p) m \llbracket a \rrbracket,(r p) m \llbracket a-1 \rrbracket+h_{1} \llbracket a-1 \rrbracket+h_{2} \llbracket a-1 \rrbracket+\cdots+h_{r} \llbracket a-1 \rrbracket\right.
$$

Since $r p=1$ and $n=m+h_{1}+h_{2}+h_{3}+\cdots+h_{r}$, the above simplifies to:

$$
n \llbracket a-1 \rrbracket
$$

And by the transitivity of the 'at least as good as' relation, we can conclude:

$$
m \llbracket a \rrbracket \preceq n \llbracket a-1 \rrbracket
$$

Which is Quantity, as desired.

The theorem can then be proved using Arrhenius's original conditions understood as adequacy conditions on population prospects and quantified over $\mathbb{W} *$. The proof is isomorphic to that given by Arrhenius (2000; forthcoming). The conclusion is as follows:

\section{The Risky First Impossibility Theorem}

There is no population prospect axiology which satisfies Egalitarian Dominance, Risky Quantity, and Quality.

\section{The Risky Second Impossibility Theorem}

Arrhenius's Second Impossibility Theorem states that the following adequacy conditions are incompatible:

Egalitarian Dominance (exact formulation): For any $a \in \mathbb{W}$, any $n \in \mathbb{N}$, and any population $X$ of size $n$ with all lives at welfare levels below $a$,

$$
X \preceq n \llbracket a \rrbracket
$$

Dominance Addition (exact formulation): For any $X$ and $Y$ of the same size with all welfare levels in $X$ higher than all welfare levels in $Y$, any $a \in \mathbb{W}$ with $a>0$, and any $m \in \mathbb{N}$,

$$
X+m \llbracket a \rrbracket \nprec Y
$$

Inequality Aversion (exact formulation): For any $a, b, c \in \mathbb{W}$ with $a>b>c$, and any $m \in \mathbb{N}$, there exists $q \in \mathbb{N}$ such that,

$$
m \llbracket a \rrbracket+q \llbracket c \rrbracket \preceq(m+q) \llbracket b \rrbracket
$$

Quality (exact formulation): There exists $m \in \mathbb{N}$ such that, for any $n \in \mathbb{N}$,

$$
n \llbracket 3 \rrbracket \preceq m \llbracket \beta \rrbracket
$$

Inequality Aversion is not particularly compelling applied to $\mathbb{W} *$. However, Risky Non-Elitism is compelling, and we saw above that Risky Non-Elitism plus 
transitivity implies that Non-Elitism is true over $\mathbb{W}^{*}$. Non-Elitism, in turn, implies Inequality Aversion:

Fix any $a, b, c, m$ as in Inequality Aversion. From Non-Elitism, we obtain corresponding $n_{1}$ : the number of lives we must raise from $c$ to $a-1$ to compensate one life falling from $a$ to $a-1$. Let $m_{1}=m$ and $q_{1}=m_{1} n_{1}$, so that $q_{1}$ gives the number of lives we must raise from $c$ to $a-1$ to compensate $m$ lives falling from $a$ to $a-1$. From Non-Elitism, we also obtain $n_{2}$ : the number of lives we must raise from $c$ to $a-2$ to compensate one life falling from $a-1$ to $a-2$, and so on. Let $m_{2}=m_{1}+q_{1}$ and $q_{2}=m_{2} n_{2}$, and so on, so that for all $i$ up to $i=a-b$ :

$$
\begin{gathered}
m_{i}=m_{i-1}+q_{i-1} \\
q_{i}=m_{i} n_{i}
\end{gathered}
$$

Consider the following population with $q=q_{1}+q_{2}+\cdots+q_{a-b}$ :

$$
m \llbracket a \rrbracket+q \llbracket c \rrbracket
$$

Since $q=q_{1}+q_{2}+\cdots+q_{a-b}$, the above can be expressed as:

$$
m \llbracket a \rrbracket+q_{1} \llbracket c \rrbracket+q_{2} \llbracket c \rrbracket+\cdots+q_{a-b} \llbracket c \rrbracket
$$

Applying Non-Elitism $m_{1}$ times yields:

$$
\preceq m \llbracket a-1 \rrbracket+q_{1} \llbracket a-1 \rrbracket+q_{2} \llbracket c \rrbracket+\cdots+q_{a-b} \llbracket c \rrbracket
$$

Applying it $m_{2}$ times yields:

$$
\preceq m \llbracket a-2 \rrbracket+q_{1} \llbracket a-2 \rrbracket+q_{2} \llbracket a-2 \rrbracket+\cdots+q_{a-b} \llbracket c \rrbracket
$$

Applying it a further $m_{3}+\cdots+m_{a-b}$ times yields:

$$
\preceq m \llbracket b \rrbracket+q_{1} \llbracket b \rrbracket+q_{2} \llbracket b \rrbracket+\cdots+q_{a-b} \llbracket b \rrbracket
$$

Since $q=q_{1}+q_{2}+\cdots+q_{a-b}$, this simplifies to:

$$
(m+q) \llbracket b \rrbracket
$$

And by the transitivity of the 'at least as good as' relation, we can conclude:

$$
m \llbracket a \rrbracket+q \llbracket c \rrbracket \preceq(m+q) \llbracket b \rrbracket
$$

Which is Inequality Aversion, as desired.

The theorem can then be proved using Arrhenius's original conditions understood as adequacy conditions on population prospects and quantified over $\mathbb{W} *$. The proof is isomorphic to that given by Arrhenius (2000; forthcoming). The conclusion is as follows:

\section{The Risky Second Impossibility Theorem}

There is no population prospect axiology which satisfies Egalitarian Dominance,

Dominance Addition, Risky Non-Elitism, and Quality. 


\section{The Risky Third Impossibility Theorem}

Arrhenius's Third Impossibility Theorem states that the following adequacy conditions are incompatible:

Egalitarian Dominance (exact formulation): For any $a \in \mathbb{W}$, any $n \in \mathbb{N}$, and any population $X$ of size $n$ with all lives at welfare levels below $a$,

$$
X \prec n \llbracket a \rrbracket
$$

Inequality Aversion (exact formulation): For any $a, b, c \in \mathbb{W}$ with $a>b>c$, and any $m \in \mathbb{N}$, there exists $q \in \mathbb{N}$ such that,

$$
m \llbracket a \rrbracket+q \llbracket c \rrbracket \preceq(m+q) \llbracket b \rrbracket
$$

Non-Sadism (exact formulation): For any $a, c \in \mathbb{W}$ with $a>0>c$, any $m, n \in \mathbb{N}$, and any population $X$,

$$
X+n \llbracket c \rrbracket \preceq X+m \llbracket a \rrbracket
$$

Non-Extreme Priority (exact formulation): There exists $n \in \mathbb{N}$ such that, for any population $X$,

$$
X+\llbracket 3 \rrbracket+n \llbracket 3 \rrbracket \preceq X+\llbracket-1 \rrbracket+n \llbracket \beta \rrbracket
$$

Quality Addition (exact formulation): For any population $X$, there exists $m \in \mathbb{N}$ such that, for any $n \in \mathbb{N}$,

$$
X+n \llbracket 3 \rrbracket \preceq X+m \llbracket \beta \rrbracket
$$

We might doubt that Inequality Aversion and Non-Extreme Priority are true over W*. However, as we saw above, Inequality Aversion follows from Risky NonElitism. Non-Extreme Priority, meanwhile, follows from Risky Non-Extreme Priority:

Risky Non-Extreme Priority (exact formulation): There exists $n \in \mathbb{N}$, and $p$ of the form $\frac{1}{r}$ with $r \in \mathbb{N}$ such that, for any $k \in \mathbb{R}$ with $0 \leq k \leq 1-p$ and any population $X$,

$$
X+[(1-k) \llbracket 3 \rrbracket,(k) \llbracket-1 \rrbracket]+n \llbracket 3 \rrbracket \preceq X+[(1-k-p) \llbracket 3 \rrbracket,(k+p) \llbracket-1 \rrbracket]+n \llbracket \beta \rrbracket
$$

These versions of General Non-Extreme Priority and Risky General Non-Extreme Priority differ only insofar as they replace welfare levels $a+1, a, b$, and $c$ with 3 , $-1,3$, and $\beta$ respectively. Therefore, the proof that Non-Extreme Priority follows from Risky Non-Extreme Priority is isomorphic to the proof that General NonExtreme Priority follows from Risky General Non-Extreme Priority given above. The theorem can then be proved using Arrhenius's original conditions understood as adequacy conditions on population prospects and quantified over $\mathbb{W}^{*}$. That proof is 
isomorphic to the one given by Arrhenius (2000; forthcoming). The conclusion is as follows:

\section{The Risky Third Impossibility Theorem}

There is no population prospect axiology which satisfies Egalitarian Dominance, Risky Non-Elitism, Non-Sadism, Risky Non-Extreme Priority, and Quality Addition.

\section{The Risky Fourth Impossibility Theorem}

Arrhenius's Fourth Impossibility Theorem states that the following adequacy conditions are incompatible:

Egalitarian Dominance (exact formulation): For any $a \in \mathbb{W}$, any $n \in \mathbb{N}$, and any population $X$ of size $n$ with all lives at welfare levels below $a$,

$$
X \prec n \llbracket a \rrbracket
$$

General Non-Extreme Priority (exact formulation): For any $a \in \mathbb{W}$, there exists $n \in \mathbb{N}$ such that, for any $b, c \in \mathbb{W}$ with $0<b \leq 3, c \geq \beta$, and any population $X$,

$$
X+\llbracket a+1 \rrbracket+n \llbracket b \rrbracket \preceq X+\llbracket a \rrbracket+n \llbracket c \rrbracket
$$

Non-Elitism (exact formulation): For any $a, c \in \mathbb{W}$ with $a-1>c$, there exists $n \in \mathbb{N}$ such that, for any population $X$ with welfare levels ranging from $c$ to $a$,

$$
X+\llbracket a \rrbracket+n \llbracket c \rrbracket \preceq X+\llbracket a-1 \rrbracket+n \llbracket a-1 \rrbracket
$$

Weak Non-Sadism (exact formulation): There exists $a \in \mathbb{W}$ with $a<0$ and $m \in \mathbb{N}$ such that, for any welfare level $b \in \mathbb{W}$ with $b>0$, any $n \in \mathbb{N}$, and any population $X$,

$$
X+m \llbracket a \rrbracket \preceq X+n \llbracket b \rrbracket
$$

Quality Addition (exact formulation): For any population $X$, there exists $m \in \mathbb{N}$ such that, for any $n \in \mathbb{N}$,

$$
X+n \llbracket 3 \rrbracket \preceq X+m \llbracket \beta \rrbracket
$$

As we saw above, General Non-Extreme Priority and Non-Elitism follow from their risky versions. The theorem can then be proved using Arrhenius's original conditions understood as adequacy conditions on population prospects and quantified over $\mathbb{W} *$. The proof is isomorphic to that given by Arrhenius (2000; forthcoming). The conclusion is as follows: 


\section{The Risky Fourth Impossibility Theorem}

There is no population prospect axiology which satisfies Egalitarian Dominance, Risky General Non-Extreme Priority, Risky Non-Elitism, Weak NonSadism, and Quality Addition.

\section{The Risky Fifth Impossibility Theorem}

Arrhenius's Fifth Impossibility Theorem states that the following adequacy conditions are incompatible:

Egalitarian Dominance (exact formulation): For any $a \in \mathbb{W}$, any $n \in \mathbb{N}$, and any population $X$ of size $n$ with all lives at welfare levels below $a$,

$$
X \prec n \llbracket a \rrbracket
$$

Dominance Addition (exact formulation): For any $X$ and $Y$ of the same size with all welfare levels in $X$ higher than all welfare levels in $Y$, any $a \in \mathbb{W}$ with $a>0$, and any $m \in \mathbb{N}$,

$$
X+m \llbracket a \rrbracket \nprec Y
$$

General Non-Elitism (exact formulation): For any $a, c \in \mathbb{W}$ with $a-1>c$, there exists $n \in \mathbb{N}$ such that, for any population $X$,

$$
X+\llbracket a \rrbracket+n \llbracket c \rrbracket \preceq X+\llbracket a-1 \rrbracket+n \llbracket a-1 \rrbracket
$$

General Non-Extreme Priority (exact formulation): For any $a \in \mathbb{W}$, there exists $n \in \mathbb{N}$ such that, for any $b, c \in \mathbb{W}$ with $0<b \leq 3, c \geq \beta$, and any population $X$,

$$
X+\llbracket a+1 \rrbracket+n \llbracket b \rrbracket \preceq X+\llbracket a \rrbracket+n \llbracket c \rrbracket
$$

Weak Quality (exact formulation): There exists $a, b \in \mathbb{W}$ with $a \geq \beta, b<0$, and $m, n \in \mathbb{N}$ such that, for any $c \in \mathbb{W}$ with $0<c \leq 3$ and any $q \in \mathbb{N}$,

$$
m \llbracket b \rrbracket+q \llbracket c \rrbracket \preceq n \llbracket a \rrbracket
$$

As we saw above, General Non-Extreme Priority follows from its risky version. The same is true of General Non-Elitism. It follows from Risky General NonElitism:

Risky General Non-Elitism (exact formulation): For any $a, c \in \mathbb{W}^{*}$ with $a-1>c$, there exists $m \in \mathbb{N}$ and $p$ of the form $\frac{1}{r}$ with $r \in \mathbb{N}$ such that, for any $k \in \mathbb{R}$ with $0 \leq k \leq 1-p$ and any population $X$,

$$
\begin{aligned}
X+[(1-k) \llbracket a \rrbracket,(k) \llbracket a-1 \rrbracket]+m \llbracket c \rrbracket \preceq & X+[(1-k-p) \llbracket a \rrbracket,(k+p) \llbracket a-1 \rrbracket] \\
& +m \llbracket a-1 \rrbracket
\end{aligned}
$$


These generalised versions of Non-Elitism and Risky Non-Elitism differ only insofar as they relax the restriction on the welfare levels contained in $X$, so the proof that General Non-Elitism follows from Risky General Non-Elitism is isomorphic to the proof that Non-Elitism follows from Risky Non-Elitism given above. The theorem can then be proved using Arrhenius's original conditions understood as adequacy conditions on population prospects and quantified over $\mathbb{W}^{*}$. That proof is isomorphic to the one given by Arrhenius (2003). The conclusion is as follows:

\section{The Risky Fifth Impossibility Theorem}

There is no population prospect axiology which satisfies Egalitarian Dominance, Dominance Addition, Risky General Non-Elitism, Risky General NonExtreme Priority, and Weak Quality.

\section{References}

Arrhenius, G. (2000). Future Generations: A Challenge for Moral Theory. PhD Thesis: Uppsala University.

Arrhenius, G. (2003). The very repugnant conclusion. In K. Segerberg \& R. Sliwinski (Eds.), Logic, Law, Morality: Thirteen Essays in Practical Philosophy in Honour of Lennart Åqvist, 29-44. Uppsala Philosophical Studies.

Arrhenius, G. (2009). One more axiological impossibility theorem. In L. G. Johansson, J. Österberg, \& R. Sliwinski (Eds.), Logic, Ethics and All That Jazz: Essays in Honour of Jordan Howard Sobel, 23-37. Uppsala Philosophical Studies.

Arrhenius, G. (2011). The impossibility of a satisfactory population ethics. In E. N. Dzhafarov \& L. Perry (Eds.), Descriptive and Normative Approaches to Human Behavior, 1-26. World Scientific Publishing Company.

Arrhenius, G. (2016). Population ethics and different-number-based imprecision. Theoria, 82(2), $166-181$.

Arrhenius, G. (forthcoming). Population Ethics: The Challenge of Future Generations. Oxford University Press.

Arrhenius, G., \& Stefánsson, H. O. (2020). Population ethics under risk. https://philpapers.org/archive/ ARRPEU.pdf.

Arrhenius, G., \& Rabinowicz, W. (2015). The value of existence. In I. Hirose \& J. Olson (Eds.), The Oxford Handbook of Value Theory, 424-44. Oxford Handbooks in Philosophy. Oxford University Press.

Asheim, G. B., \& Zuber, S. (2016). Evaluating intergenerational risks. Journal of Mathematical Economics, 65, 104-117.

Blackorby, C., Bossert, W., \& Donaldson, D. (2005). Population Issues in Social Choice Theory, Welfare Economics, and Ethics. Cambridge University Press.

Blackorby, C., \& Donaldson, D. (1991). Normative population theory: A comment. Social Choice and Welfare, 8(3), 261-267.

Broome, J. (2004). Weighing Lives. Oxford University Press.

Budolfson, M., \& Spears, D. (2018). Why the repugnant conclusion is inescapable. https://scholar. princeton.edu/sites/default/files/cfi/files/budolfson_spears_2018_repugnant_cfi.pdf.

Bykvist, K. (2007). The good, the bad and the ethically neutral. Economics \& Philosophy, 23(1), 97-105.

Carlson, E. (1998). Mere addition and two trilemmas of population ethics. Economics \& Philosophy, 14(2), 283-306.

Carlson, E. (forthcoming). On some impossibility theorems in population ethics. In G. Arrhenius, K. Bykvist, T. Campbell, \& E. Finneron-Burns (Eds.), The Oxford Handbook of Population Ethics. Oxford University Press.

Chang, R. (2016). Parity, imprecise comparability, and the repugnant conclusion. Theoria, 82(2), $183-215$. 
Crisp, R. (1997). Mill on Utilitarianism. Routledge.

Crisp, R. (2006). Reasons and the Good. Oxford University Press.

Griffin, J. (1988). Well-Being: Its Meaning, Measurement and Moral Importance. Oxford University Press.

Handfield, T., \& Rabinowicz, W. (2018). Incommensurability and vagueness in spectrum arguments: Options for saving transitivity of betterness. Philosophical Studies, 175(9), 2373-2387.

Kitcher, P. (2000). Parfit's puzzle. Noûs, 34(4), 550-577.

McTaggart, J. M. E. (1927). The Nature of Existence Volume II. Cambridge University Press.

Mill, J. S. (1861). Utilitarianism. In R. Crisp (Ed.). Oxford University Press. 1998.

Moore, G. E. (1903). Principia Ethica. In T. Baldwin (Ed.). Cambridge University Press. 1993.

Nebel, J. M. (2017). Priority, not equality, for possible people. Ethics, 127(4), 896-911.

Nebel, J. M. (2019). An intrapersonal addition paradox. Ethics, 129(2), 309-343.

Nebel, J. M. (2021). Totalism without repugnance. In J. McMahan, T. Campbell, J. Goodrich, \& K. Ramakrishnan (Eds.), Ethics and Existence: The Legacy of Derek Parfit. Oxford University Press. https://philpapers.org/archive/NEBTWR.pdf.

Ng, Y. -K. (1989). What should we do about future generations? Economics \& Philosophy, 5(2), 235-253.

Parfit, D. (1984). Reasons and Persons. Clarendon Press.

Rachels, S. (2004). Repugnance or intransitivity: A repugnant but forced choice. In J. Ryberg \& T. Tännsjö (Eds.), The Repugnant Conclusion: Essays on Population Ethics. Kluwer Academic Publishers.

Roberts, M. A. (2007). The non-identity fallacy: Harm, probability and another look at Parfit's depletion example. Utilitas, 19(3), 267-311.

Spears, D., \& Budolfson, M. (2019). Why variable-population social orderings cannot escape the repugnant conclusion: Proofs and implications. http://ftp.iza.org/dp12668.pdf.

Tännsjö, T. (2002). Why we ought to accept the repugnant conclusion. Utilitas, 14(3), 339-359.

Temkin, L. S. (2012). Rethinking the Good: Moral Ideals and the Nature of Practical Reasoning. Oxford University Press.

Thomas, T. (2016). Topics in Population Ethics. DPhil Thesis: University of Oxford.

Thomas, T. (2018). Some possibilities in population axiology. Mind, 127(507), 807-832.

Thornley, E. (forthcoming). A dilemma for lexical and Archimedean views in population axiology. Economics \& Philosophy.

Publisher's Note Springer Nature remains neutral with regard to jurisdictional claims in published maps and institutional affiliations. 